International Journal of Wireless \& Mobile Networks (IJWMN) Vol. 5, No. 1, February 2013

\title{
A NOVEL APPROACH FOR HYBRID OF ADAPTIVE AMPLITUdE NON-LINEAR GRADIENT DECENT (AANGD) AND COMPLEX LEAST MEAN SQUARE (CLMS) ALGORITHMS FOR SMART ANTENNAS
}

\author{
Y. Rama Krishna ${ }^{1}$ P.V. Subbaiah ${ }^{2}$ B. Prabhakara Rao ${ }^{3}$ \\ ${ }^{1}$ Prasad V. Potluri Siddhartha Institute of Technology, Vijayawada, India \\ ramakrishna.yarlagadda@gmail.com \\ ${ }^{2}$ Amrita Sai Institute of Science \& Technology, Vijayawada, India \\ pvs_ece2000@yahoo.co.in \\ ${ }^{3}$ JNT University Kakinada, Kakinada, India \\ drbprarediffmail.com
}

\begin{abstract}
An adaptive beam former is a device, which is able to steer and modifies an array's beam pattern in order to enhance the reception of a desired signal, while simultaneously suppressing interfering signals through complex weight selection. However, the weight selection is a critical task to get the low Side Lobe Level (SLL) and Low Beam Width. One needs to have a low SLL and low beam width to reduce the antenna's energy radiation/reception ability in unintended directions. The weights can be chosen to minimize the SLL and to place nulls at certain angles. The convergence of the array output towards desired signal is also very important for a good signal processing tool of an adaptive beam former. A vast number of possible window functions are available to calculate the weights for Smart Antennas. From the analysis of many of these algorithms, it is observed that there is a compromise between HPBW and SLL. But in case of smart antennas, both of these parameters must have low values to get good performance. In our earlier work it is proposed that Complex Least Mean Square (CLMS) and Augmented Complex Least Mean Square ( ACLMS) algorithms gives low beam width and side lobe level in noisy environment. Another neural algorithm Adaptive Amplitude Non Linear Gradient Decent algorithm (AANGD) has the advantage of more number of control parameters over CLMS and ACLMS algorithms. In this paper the hybrid of CLMS and AANGD is presented and this novel hybrid algorithm has outperformed the hybrid algorithm of CLMS and ACLMS in the aspect of convergence towards the desired signal.
\end{abstract}

\section{KEYWORDS}

AANGD, CLMS, ACLMS, Beam forming, Smart Antennas, Adaptive Beam former

\section{INTRODUCTION}

Wireless cellular networks are fast growing technology in the current world and this trend is likely to continue for several years [1]. The advancements in radio technology enable novel and improved services in the cellular systems. Current wireless services include transmission of voice, fax, and multimedia applications and so on. Multimedia services like video-on demand and internet access needs more band width. Wireless networks must provide these services in a wide range of environments, spanning dense urban, suburban, and rural areas.

Smart Antennas (SA) [2,3] consist of an array of antenna elements with signal processing capability that optimizes the radiation and reception of a desired signal dynamically. SAs can place nulls in the direction of interferers via adaptive updating of weights linked to each antenna element. SAs, thus cancel out most of the co-channel interference resulting the better quality of 
reception and lowered dropped calls. SAs can also track the user within a cell via direction of arrival algorithms.

The Smart antennas $[4,5]$ perform spatial filtering, which makes it possible to receive energy from a particular direction, while simultaneously blocking it from another direction. This property makes smart antennas as an effective tool in detecting and locating radiation from other sources. That means, the design and development of the efficient models to this task for real time optimization is a current problem. The control unit of the Smart Antenna is normally realized with a Digital Signal Processing (DSP) unit. Based on certain inputs, the DSP algorithm controls radiation parameters of the antenna to optimize communication link. Figure 1 shows the basic model of Smart Antenna (SA).

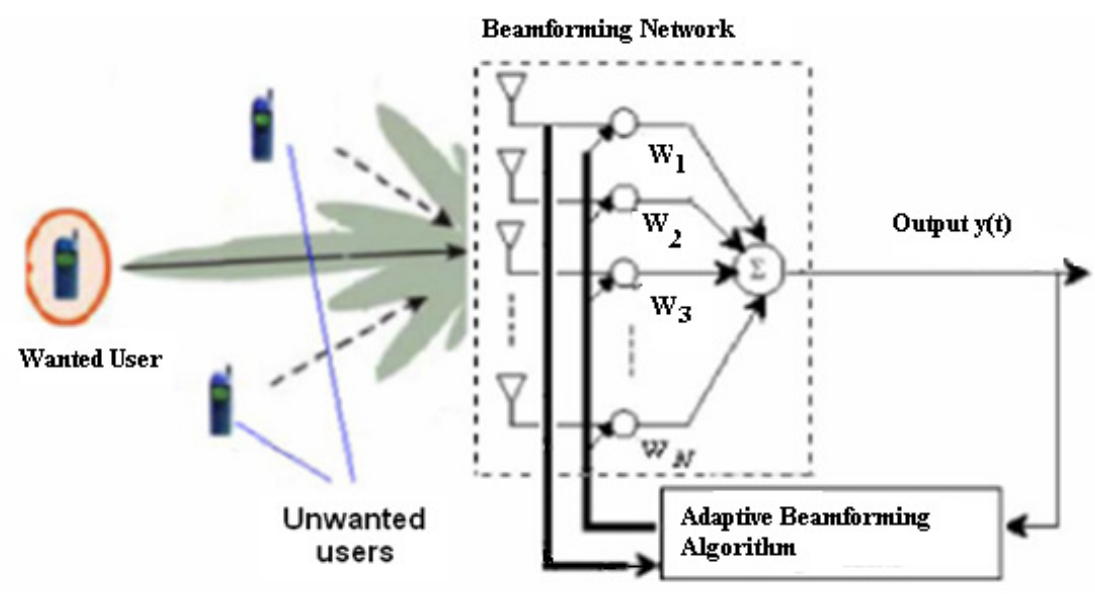

Figure 1. Basic smart antenna system

\section{COMPLEX NEURAL ALGORITHMS}

A Complex Valued Neural Network [6, 7, 12] is an artificial neural network, consists of complex valued input signals, weights, threshold values and/or signal functions. Such kind of models must be needed for solving the problems in the field of signal processing. In the signal processing, signals are complex valued and processing of such signals requires the implementation of new complex valued neural processing models. One of the most important characteristics of the complex valued neural models is their ability to process linear complex signals of the smart antennas. In smart antennas signals from different sources or interferers are to be processed before orienting the main beam direction of the antenna array. In this context identifying the angle of arrival of the desired signal is very important. More over the Half Power Beam Width (HPBW) of the array radiation pattern must be as small as possible to avoid the interference, similarly the Side Lobe Level (SLL).

In this paper CLMS and AANGD algorithms $[2,6,8]$ have been considered as complex valued neural networks that can be applied on complex signals of Smart Antenna System.

Least Mean Square (LMS) is fundamental gradient based algorithm introduced by Widrow and Hoff in 1959 that estimates the gradient vector from the available data. This algorithm is an iterative method that leads to Minimum Mean Square Error (MMSE), but this is a simple model which cannot process complex data with more noise. In the analysis of LMS, it is observed that, its convergence is slow if the Eigen values are widely spread and it is directly depends on the Eigen structure. The convergence time of LMS can be exceedingly long and highly data dependent when the Eigen values of the covariance matrix differs. 
In order to process such complex signals, variant of LMS models such as CLMS and ACLMS algorithms were used in noiseless environment and from the results it is observed that CLMS has an edge over ACLMS algorithm in both HPBW and SLL, where as in case of convergence towards the desired signal, the performance of ACLMS is better than CLMS algorithm.

When the performance of these two algorithms is considered in real time environment, i.e. noisy environment, at low values of step size parameter $(\mu)$, ACLMS algorithm has given good performance in both HPBW and SLL. Hence it is observed that CLMS is suitable for low noise environments and ACLMS is suitable for noisy environments.

However in CLMS and ACLMS algorithm there is no control over adaptation of HPBW and SLL. As soon as the $\mu$ value and $\mathrm{N}$ value changes, the HPBW and SLL values changes automatically and one can't expect a particular value for the above parameters. This problem is addressed in this paper by making use of the Adaptive amplitude Nonlinear Gradient Decent (AANGD) algorithm in which two more parameters, initial value of non linearity $(\lambda)$ and step size of adaptive amplitude ( $\rho)$ are included in the model [1]. The HPBW and SLL can be controlled and required values for these two can be obtained. The values are presented in the table no 1.

\subsection{Complex Least Mean Square Algorithm (CLMS)}

CLMS $[9,10]$ algorithm was introduced by Widrow et al. in 1975, which benefits from the robustness and stability of the LMS and enables the simultaneous processing of complex signals.

This algorithm performs stochastic gradient decent in complex domain statistics that enables better modeling of complex data and produce effective outcome. The basic algorithm of CLMS is discussed in [2]. The weight update and the output of the CLMS algorithm is as follows:

1. The stochastic gradient adaptation for the weight vector can be expressed as

$$
\mathrm{w}(\mathrm{k}+1)=\mathrm{w}(\mathrm{k})+\mu \mathrm{e}(\mathrm{k}) \mathrm{x}^{*}(\mathrm{k}), \mathrm{w}(0)=0
$$

2. This output of the CLMS algorithm is computed as

$$
y=x^{H}(k) w(k) \rightarrow w(k+1)=w(k)+\mu e(k) \mathrm{x}^{*}(\mathrm{k})
$$

\subsection{Adaptive Amplitude Nonlinear Gradient Decent Algorithm (AANGD)}

Based on the standard weight update, adaptive learning rates $\mathrm{n}(\mathrm{k})$ for the CLMS, normalized CLMS, and normalized adaptive nonlinear gradient decent (ANGD) algorithms $[9,10,11]$ are given by

$$
n(k)=\begin{array}{ll}
\mu & \text { for CLMS } \\
\frac{\mu}{|x(k)|_{2}^{2}+\varepsilon} & \text { for CNLMS } \\
\frac{\mu}{|\emptyset(k)|^{2}|x(k)|_{2}^{2}+\varepsilon} & \text { for ANGD }
\end{array}
$$

Where $\varepsilon$ is a regularization parameter used to prevent divergence for close to zero inputs. The class of ANGD algorithm performs 'linear' updates which depend upon estimators of $\frac{\partial J(k)}{\partial \mu}$. This is achieved by making the step size (amplification factor) within the weight update gradient adaptive [11, 12]. These algorithms are based on two coupled unconstrained optimization procedures (for weights and step size), which can affect their robustness to the initial values of the parameters.

The basic algorithm of ANGD is as follows: 


$$
\begin{aligned}
& e(k)=d(k)-\emptyset\left(x^{T}(k) w(k)\right) \\
& \emptyset\left(x^{T}(k) w(k)\right)=\lambda(k) \bar{\emptyset}\left(x^{T}(k) w(k)\right) \\
& w(k+1)=w(k)+n(k) e(k) \emptyset^{j *}\left(\left(x^{T}(k) w(k) x^{*}(k)\right)\right. \\
& n(k)=\frac{\mu}{\mid \emptyset^{\prime}\left(\left(\left.x^{T}(k) w(k)\right|^{2}|x(k)|_{2}^{2}+\varepsilon(k)\right.\right.}
\end{aligned}
$$

Where $\varepsilon(k)$ is an adaptive regularization parameter [1]. Based on the cost function

$$
\begin{aligned}
& J(k)=\frac{1}{2} e(k) e^{*}(k)=\frac{1}{2}|e(k)|^{2} \\
& \lambda(k+1)=\lambda(k)+\frac{\rho}{2}\left|e^{*}(k) \bar{\emptyset}\left(x^{T}(k) w(k)\right)+e(k) \overline{\emptyset^{*}}\left(x^{T}(k) w(k)\right)\right|
\end{aligned}
$$

\subsection{Hybrid Model}

In the Hybrid Model, CLMS and AANGD have been combined since each algorithm has its own merits and demerits in the process of adaptive beam forming signals in Smart Antennas. In CLMS, even though the array output signal convergence towards the desired signal is good the control of HPBW vs SLL is poor. Similarly in AANGD, even though array output signal convergence towards the desired signal is poor the control of HPBW vs SLL is good with more number of control parameters that can be discussed in section 3 (figure 2-4 and table 1).

In the analysis of CLMS and AANGD with experimental results, the following model proposed combining these two algorithms as hybrid which is often referred as Hybrid of CLMS and $\boldsymbol{A A N G D}$. The hybrid algorithm is as follows:

$$
\begin{aligned}
& y_{C L M S}(k)=x^{H}(k) w_{C L M S}(k) \rightarrow w_{C L M S}(k+1)=w(k)+\mu_{1} e(k) x(k) \\
& y_{A A N G D}(\mathrm{k})=\mathrm{h}^{\mathrm{T}}(\mathrm{k}) \mathrm{z}(\mathrm{k})+\mathrm{g}^{\mathrm{T}}(\mathrm{k}) \mathrm{z}^{*}(\mathrm{k}) \\
& h(k+1)=h(k)+\mu_{2} e(k) z^{*}(k) \\
& g(k+1)=g(k)+\mu_{2} e(k) z(k) \\
& e_{C L M S}(k)=d(k)-x^{T}(k) w_{C L M S}(k) \\
& e_{A A N G D}(k)=d(k)-z^{a T}(k) w_{A A N G D}(k) \\
& \mathrm{Y}_{\text {Hybrid }}=\lambda^{*} \mathrm{Y}_{\text {CLMS }}(\mathrm{k})+(1-\lambda)^{*} \mathrm{Y}_{\text {AANGD }}(\mathrm{k}) \\
& \mathrm{e}_{\text {Hybrid }}(\mathrm{k})=\mathrm{x}-\mathrm{Y}_{\text {Hybrid }}(\mathrm{k}) \\
& \mathrm{W}_{\text {Hybrid }}(\mathrm{k}+1)=\lambda(\mathrm{k}) \mathrm{W}_{\text {CLMS }}(\mathrm{k})+(1-\lambda) \mathrm{W}_{\text {ACLMS }}(\mathrm{k})^{*} \\
& \lambda(\mathrm{k}+1)=\lambda(\mathrm{k})+\mu 3^{*} \text { real }\left(\mathrm{e}_{\text {Hybrid }}(\mathrm{k})^{*}\left(\mathrm{Y}_{\text {CLMS }}(\mathrm{k})-\mathrm{Y}_{\text {AANGD }}(\mathrm{k})\right)\right.
\end{aligned}
$$

In Algorithm 1, 2 \& 3 [2, 4, 5, 6], some notations have been considered for step size parameters such as $\mu 1$ for $C L M S, \mu 2$ for $A A N G D, \mu 3$ for Hybrid and $\lambda$ for mixing parameter.

The following constraints are to be considered while constructing the Hybrid model for the combination of CLMS and AANGD.

1. When the $\mu$ values are considered for these algorithms, It needs to satisfy the following conditions for step size parameters

$$
\mu 3>\mu 1>\mu 2 \text { and } \mu 3=0.8 .
$$

2. If $\mu 1$ and $\mu 2$ are considered the same values, CLMS and AANGD gives the best performance for HPBW and SLL but in the case of Hybrid it cannot.

3. If $\mu$ values are reduced to low levels the convergence of array output towards desired signal for CLMS and AANGD is satisfactory but when the $\mu$ values are low, the convergence towards desired signal will be too poor, which causes over damped condition. 
International Journal of Wireless \& Mobile Networks (IJWMN) Vol. 5, No. 1, February 2013

\section{PERFORMANCE ANALYSIS}

The simulations are carried out with an input signal $x_{s}(k)=\cos (2 w t)$ at frequency of $1 \mathrm{kHz}$ along with a random noise. In order to simulate the real time environment of Smart Antenna System, the noise component has been considered in addition to the input signal and the performance of the CLMS and AANGD algorithms have been analysed with different values of $\mathrm{N}$ and $\mu$. When the step size parameter value ' $\mu$ ' is reduced, there is an improvement in the performance of the chosen algorithms in noisy environment rather than noiseless scenario [2, 4, $6]$. When a random noise is added, the behavior of the algorithm is completely changed and the error convergence requires more number of iterations and this process is time consuming. Moreover the performance of ACLMS also indicate that either upward or downward trend in HPBW cannot be predicted in one direction [3]. Even though low HPBW and SLL are observed in noisy environment at very low value of $\mu$, the trend of the results is not consistent.

The results obtained with ANGD algorithm and its comparison with CLMS algorithms in noisy environment is presented in table 1. From this comparison it is observed that, as the initial value of step size of adaptive amplitude $(\rho)$ is fixed at 0.001 and $\lambda$ value is decreased, a reduction in both HPBW and SLL are observed which is a very interesting trend and was not observed in earlier neural algorithms.

Table1. Performance comparison of ANGD algorithm with CLMS \& ACLMS Algorithms

\begin{tabular}{|c|c|c|c|c|c|c|}
\hline Algorithm & $\begin{array}{c}\text { No. of array } \\
\text { elements } \\
\mathbf{N}\end{array}$ & $\begin{array}{c}\text { Step Size } \\
\mu\end{array}$ & $\begin{array}{c}\text { Step size of } \\
\text { adaptive } \\
\text { amplitude } \\
\rho \\
\end{array}$ & $\begin{array}{c}\text { Initial value of } \\
\text { amplitude of } \\
\text { non linearity } \\
\lambda\end{array}$ & $\begin{array}{c}\text { Half Power } \\
\text { Beam Width } \\
(\text { HPBW) }\end{array}$ & $\begin{array}{c}\text { Side Lobe } \\
\text { Level } \\
\text { (SLL) }\end{array}$ \\
\hline CLMS & 8 & 0.002 & -- & -- & 6.6 & 0.1555 \\
\hline \multirow{5}{*}{ AANGD } & \multirow{5}{*}{8} & \multirow{5}{*}{0.002} & \multirow{5}{*}{0.001} & 0.9 & 7.4 & 0.2140 \\
\hline & & & & 0.6 & 7.1 & 0.2030 \\
\hline & & & & 0.3 & 7.0 & 0.1967 \\
\hline & & & & 0.25 & 6.7 & 0.1555 \\
\hline & & & & 0.2 & 6.4 & 0.1445 \\
\hline
\end{tabular}

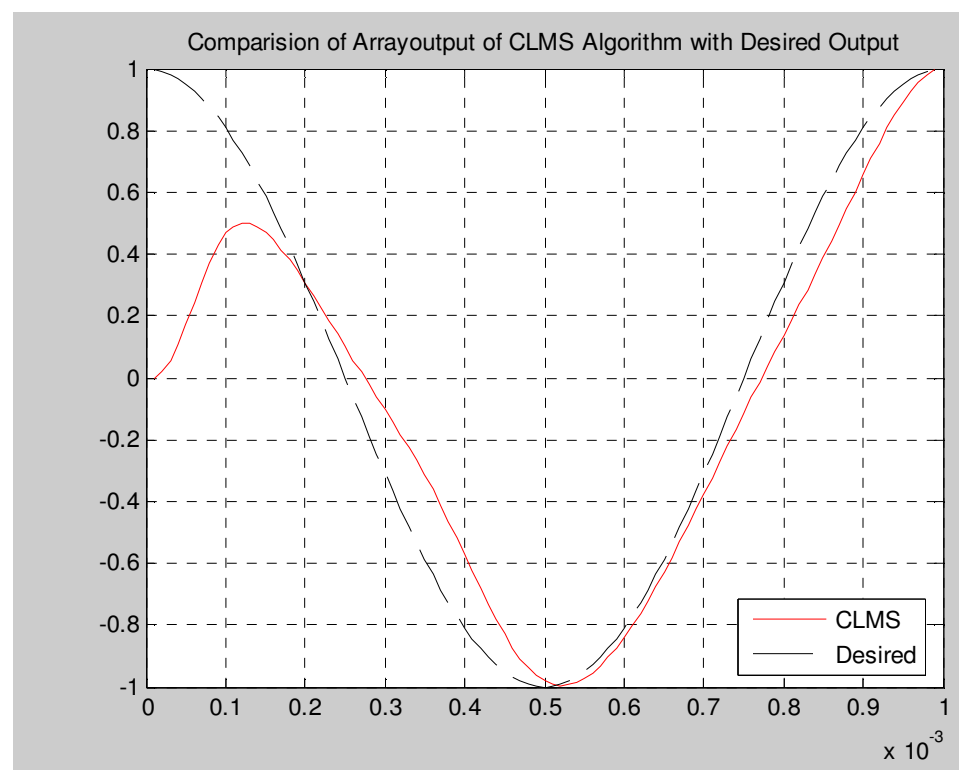

Figure 2. Comparison of Array output generated using CLMS algorithm with desired signal 
International Journal of Wireless \& Mobile Networks (IJWMN) Vol. 5, No. 1, February 2013

The advantage of CLMS algorithm over AANGD is its fast convergence towards the desired signal. Hence to make use of the individual best aspects of these algorithms, a hybrid model of these two is designed and tested with same input and desired signals. This novel hybrid algorithm has outperformed the hybrid algorithm of CLMS and ACLMS in the aspect of convergence towards the desired signal.

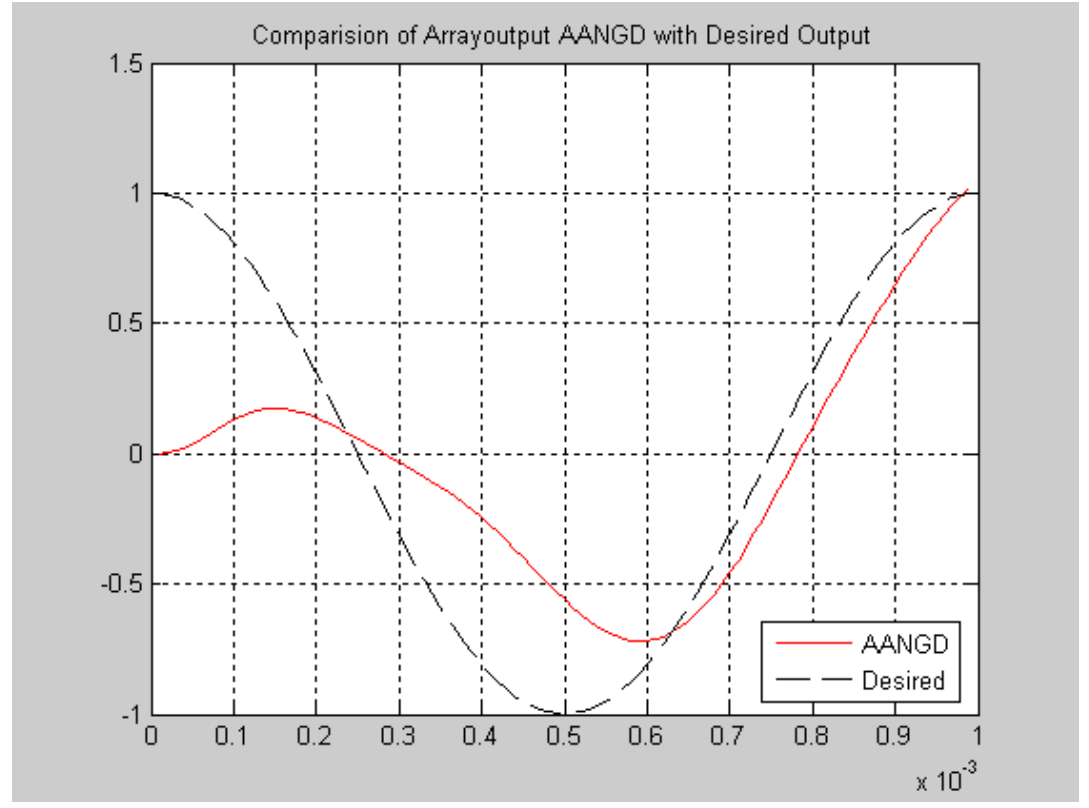

Figure 3. Comparison of Array output generated using AANGD algorithm with desired signal

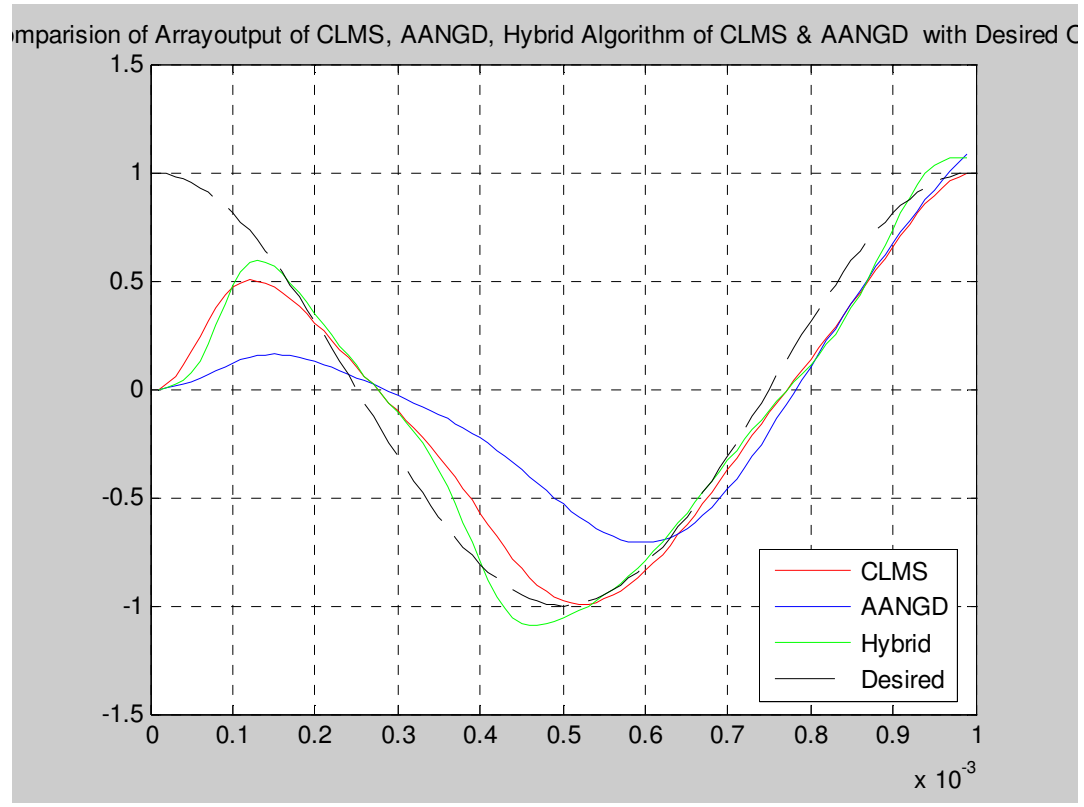

Figure 4. Comparison of Array outputs generated using CLMS, AANGD and Hybrid algorithm with desired signal 
International Journal of Wireless \& Mobile Networks (IJWMN) Vol. 5, No. 1, February 2013

\section{CONCLUSION}

Adaptive nonlinear gradient decent algorithm is considered along with complex valued neural network like CLMS on adaptive beam forming signals in Smart Antennas [2] with various parameters such as number of array elements $(\mathrm{N})$, learning rate $(\mu)$, initial value of non linearity $(\lambda)$, step size of adaptive amplitude $(\rho)$ have been considered under noiseless and noisy environments. From the analysis of [2], CLMS is a better model than AANGD in the convergence of desired signal, but AANGD is better model than CLMS in good control over adaptation of these two parameters with $\lambda$ and $\rho$ with similar performance as observed in CLMS and ACLMS in noisy and noiseless environments. Such algorithms are beneficial when dealing with signals that have rich dynamical behavior. In order to improve the overall performance of Smart Antenna System by making use of the individual best aspect of CLMS, AANGD algorithms, a new approach is proposed by combining these models as hybrid which has improved the performance further. Similarly on the same grounds a hybrid of CLMS and AANGD models may be proposed for further improvement.

\section{REFERENCES}

[1] Andrew I. Hanna and D. P. Mandic, "A complex-valued nonlinear neural adaptive filter with a gradient adaptive amplitude of the activation function", Journal of Neural Networks, vol. 16, issue 2, pp. 155-159, 2003.

[2] Y. Ramakrishna, PESN Krishna Prasad, P.V. Subbaiah and B. Prabhakara Rao, "A Performance Analysis of CLMS and Augmented CLMS Algorithms for Smart Antennas ", Proc. $4^{\text {th }}$ International Workshop on Computer Networks and Communications, Coimbatore 2012, pp. 9-19, DOI: 10.5121/csit.2012.2402.

[3] Jack H. Winters, "Smart Antennas for Wireless Systems", IEEE Personal Communications, Vol. 5, No. 1, pp. 23-27, 1998.

[4] Y. Ramakrishna, PESN Krishna Prasad, P.V. Subbaiah and B. Prabhakara Rao, "A Hybrid Model of CLMS and ACLMS Algorithms for Smart Antennas", Proc. $4^{\text {th }}$ International Conference on Networks and Communications (NetCom2012) published by Springer-Verlag New York. Inc., December 2012.

[5] L.C. Godara, "Applications of Antenna Arrays to Mobile Communications. I. Performance Improvement, Feasibility and Considerations”, IEEE Proceedings, Vol. 85, No.7, pp. 1031-1060, 1997.

[6] Y. Ramakrishna, P.V. Subbaiah, B. Prabhakara Rao and PESN Krishna Prasad, "Adaptive Non-linear Gradient Decent Algorithm for Smart Antennas", International Journal of Mobile Network Communications \& Telematics (IJMNCT), Vol. 2, No. 6, December 2012. pp. 11-19, DOI: 10.5121/ijmnct.2012.2602.

[7] Smart Antennas - Beamforming Tutorial”, www.altera.com

[8] Akira Hirose, "Complex valued Neural Networks: Theories and Applications", World Scientific Publications, 2003.

[9] Soroush Javidi, Maciej Pedzisz, Su Lee Goh and Danilo P. Mandic, "The Augmented Complex Least Mean Square Algorithm With Application to Adaptive Prediction Problems", Proc. 1st IARP Workshop on Cognitive Information Processing, 2008, 54-57.

[10] D. P. Mandic, Yili Xia and S. C. Douglas, "Steady State Analysis of the CLMS and Augmented CLMS Algorithms for Non-Circular Complex Signals", Proceedings of ASILOMAR, 2010, pp. 1635-1639.

[11] D. P. Mandic, Yili Xia and Ali H Syad, "An Adaptive Diffusion Augmented CLMS algorithm for Distributed Filtering of Non-Circular Complex Signals”, IEEE Signal Processing Letters, Vol. 18, No. 11, 2011.

[12] D. P. Mandic and Vanessa Su Lee Goh, "Complex Valued Nonlinear Adaptive Filters Noncircularity, Widely Linear and Neural Models”, John Wiley \& Sons Ltd., 2009. 
International Journal of Wireless \& Mobile Networks (IJWMN) Vol. 5, No. 1, February 2013

Authors

Y. Ramakrishna currently pursuing Ph.D. Degree from JNTU Kakinada in the field of Smart Antennas for Mobile Communications. He received M.Tech Degree in Microwave Engineering from Acharya Nagarjuna University, India in 2005. Currently He is working as Associate Professor in the Department of ECE, Prasad V. Potluri Siddhartha Institute of Technology, India. He is also a Member of ISTE. His Research interest includes Smart Antennas, Antennas and Wave Propagation, Mobile Communications and Microwave Engineering.

Dr. P. V. Subbaiah received his Ph.D. in Microwave Antennas from JNTU, India, 1995. His Master's Degree in Control Systems from Andhra University, India, 1982. $\mathrm{He}$ is Currently Working as Principal at Amrita Sai Institute of Science and Technology, Vijayawada, India since 2007. His Research interest includes Microwave Antennas, Optical Communications and Mobile Communications.
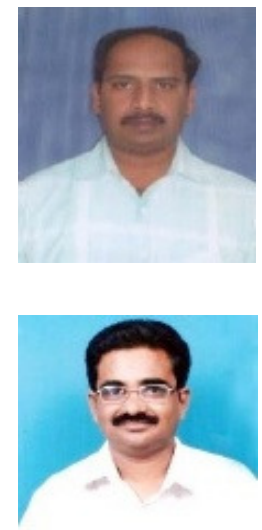

Dr. B. Prabhakara Rao received his Ph.D. from IISc Bangalore, India. He is currently working as professor in the department of Electronics and Communication Engineering. He has more than 30 years of experience in teaching and 24 years of $\mathrm{R}$ $\& \mathrm{D}$. He is an expert in signal processing \& Communications. He produced $7 \mathrm{PhD}$ 's and guiding 20 Phd scholars. He held different positions in his career like head of the department, vice principal, in JNTU College of engineering, Director (Institute of Science \& Technology), Director of Evaluation and Director (Foreign Universities and Alumni relations) in JNT University from 2003. He published more

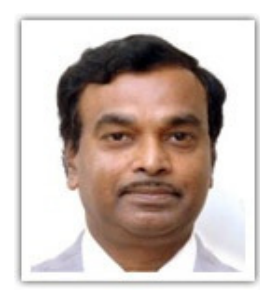
than 120 technical papers in national and international journals and conferences. His areas of interests are Wireless Communications, Digital Communications, Image Processing, Optical Networks, Network Security etc. 